\title{
BMJ
}

\section{Parenteral dexamethasone for acute severe migraine headache: meta-analysis of randomised controlled trials for preventing recurrence}

\author{
Ian Colman, assistant professor, ${ }^{1}$ Benjamin W Friedman, assistant professor, emergency physician, ${ }^{2}$ \\ Michael D Brown, professor, ${ }^{3}$ Grant D Innes, professor, head, ${ }^{4}$ Eric Grafstein, emergency physician, ${ }^{5}$ \\ Ted E Roberts, assistant professor, director, ${ }^{6}$ Brian H Rowe, professor, research director ${ }^{1,7}$
}

${ }^{1}$ School of Public Health, University of Alberta, Edmonton, $A B$, Canada

${ }^{2}$ Department of Emergency Medicine, Albert Einstein College of Medicine, Bronx, NY, USA

${ }^{3}$ Department of Epidemiology and Program in Emergency Medicine, Michigan State University, Grand Rapids, MI, USA

${ }^{4}$ Division of Emergency Medicine, University of Calgary, AB, Canada ${ }^{5}$ Department of Emergency Medicine, Providence Health Care and St Paul's Hospital, Vancouver, $\mathrm{BC}$, Canada

${ }^{6}$ Division of Neurology, Department of Medicine, University of Alberta, Edmonton, AB, Canada

${ }^{7}$ Department of Emergency Medicine, University of Alberta, 1G1.42 Walter C Mackenzie Centre, 8440-112 Street

Edmonton, AB, Canada T6G 2B7 Correspondence to: $\mathrm{B} H$ Rowe brian.rowe@ualberta.ca

doi:10.1136/bmj.39566.806725.BE

\section{ABSTRACT}

Objective To examine the effectiveness of parenteral corticosteroids for the relief of acute severe migraine headache and prevention of recurrent headaches.

Design Meta-analysis.

Data sources Electronic databases (Cochrane Central Register of Controlled Trials, Medline, Embase, LILACS, and $\mathrm{CINAHL),} \mathrm{conference} \mathrm{proceedings,} \mathrm{clinical} \mathrm{practice}$ guidelines, contacts with industry, and correspondence with authors.

Selection criteria Randomised controlled trials in which corticosteroids (alone or combined with standard abortive therapy) were compared with placebo or any other standard treatment for acute migraine in adults.

Review methods Two reviewers independently assessed relevance, inclusion, and study quality. Weighted mean differences and relative risks were calculated and are reported with $95 \%$ confidence intervals.

Results From 666 potentially relevant abstracts, seven studies met the inclusion criteria. All included trials used standard abortive therapy and subsequently compared single dose parenteral dexamethasone with placebo, examining pain relief and recurrence of headache within 72 hours. Dexamethasone and placebo provided similar acute pain reduction (weighted mean difference 0.37 , $95 \%$ confidence interval -0.20 to 0.94 ). Dexamethasone was, however, more effective than placebo in reducing recurrence rates (relative risk $0.74,95 \%$ confidence interval 0.60 to 0.90$)$. Side effect profiles between dexamethasone and placebo groups were similar. Conclusion When added to standard abortive therapy for migraine headache, single dose parenteral dexamethasone is associated with a $26 \%$ relative reduction in headache recurrence (number needed to treat=9) within 72 hours.

\section{INTRODUCTION}

Migraine is a common and disabling health problem among adults. Surveys from around the world suggest that $6 \%$ of men and $15-17 \%$ of women experience migraine headaches. ${ }^{1}$ These headaches cause major disability, and in the United States the annual costs of lost productivity range from $\$ 5.6 \mathrm{bn}(£ 2.9 \mathrm{bn} ; € 3.6 \mathrm{bn})$ to $\$ 17 \mathrm{bn} .^{2}$

People with acute severe migraine often present to the emergency department; about $5 \%$ of visits to an emergency department are for migraine headache. ${ }^{3}$ Many agents are used to treat acute severe migraine. Clinical guidelines recommend sumatriptan, dihydroergotamine, ergotamine, chlorpromazine, prochlorperazine, and several others. ${ }^{4-7}$ These agents are moderately effective in relieving acute migraine (about $60-70 \%)^{8}$; it is, however, common for migraine to recur within 24 to 72 hours, often of equal or greater severity to the index episode. One study reported that $49 \%$ of people treated for migraine headache in the emergency department had a recurrent headache within 24 hours, ${ }^{9}$ which is distressing for patients and problematic for overcrowded emergency departments. ${ }^{10}$

Many factors are likely to underlie the pathophysiology of migraine, including neuronal hyperexcitability, activation of the trigeminovascular system, and possibly an inflammatory process. ${ }^{11-13}$ Therefore moderating the inflammatory cascade may help to relieve the migraine headache and prevent its recurrence. A variety of anti-inflammatory agents may provide benefit through the mitigation of inflammation. Corticosteroids and non-steroidal anti-inflammatory drugs are the most appealing, economical, and familiar agents with which to test this hypothesis of antiinflammatory therapy. The use of corticosteroids in the emergency department might vary; however, published reports suggest that corticosteroids are infrequently used for acute severe migraine. ${ }^{3}$

We assessed the evidence from controlled trials on the effectiveness and tolerability of parenteral corticosteroids for the relief of acute migraine headache in adults and the prevention of recurrences.

\section{METHODS}

Our study protocol was developed to outline the search strategy, to establish explicit trial selection criteria, to clarify the data abstraction process, and to define the analysis. $^{14}$ 
We searched the Cochrane Central Register of Controlled Trials, Medline, Embase, LILACS, and CINAHL using the terms "headache" or "migraine" and several terms to identify corticosteroids: prednisone OR methylprednisolone OR hydrocortisone OR dexamethasone OR decadron OR triamcinolone OR betamethasone OR prednisolone OR solucortef OR solu-cortef. Terms were exploded whenever possible within each database. Because of the small number of relevant abstracts we eliminated any restrictions for randomised studies from the database searches to produce a more sensitive search. In Embase the search required that a form of the word "random" had to appear in the title or abstract, and forms of the words "intravenous" or "intramuscular" had to appear in the text.

In addition to searching electronic databases we hand searched conference proceedings from major meetings on neurology, headache, and emergency medicine for the past 10 years; reviewed clinical practice guidelines for the management of acute migraine; searched websites housing clinical trial details, theses, or dissertations; and contacted pharmaceutical companies, authors of previously identified studies, and experts in headache. Finally, we searched the reference lists of potentially relevant studies to identify additional studies. Language of publication and non-publication were not reasons for exclusion.

Eligible studies for review were randomised controlled trials of parenteral corticosteroids given for acute severe migraine in adults ( 18 years or older) along with reasonable criteria to distinguish migraine from other headache types. We included trials if they were done only in a setting that indicated the migraine was an acute and severe event (emergency department or headache clinic). Parenteral delivery was chosen because most treatments for migraine headache in the emergency department are delivered intravenously or intramuscularly, to counter the nausea and vomiting often associated with migraine. ${ }^{3}$

The primary outcome considered was recurrence of migraine headache within 24 to 72 hours of treatment. To meet the definition of recurrent migraine we required that the original migraine must have largely resolved. Not all trials tackled this specifically; however, as all eligible trials occurred in emergency departments or headache clinics we assumed that patients would not have been discharged without substantial relief from their original migraine. Secondary outcomes considered were reduction in headache pain after treatment on a $10 \mathrm{~cm}$ visual analogue scale, and adverse events associated with the treatment.

Two independent reviewers (IC and BHR) screened the titles and abstracts of eligible studies. Potentially relevant papers were obtained and two independent reviewers (IC and $\mathrm{MDB}$ ) reviewed the full manuscripts for possible inclusion. Disagreements were resolved by consensus. Information on patients, methods, interventions, outcomes, and adverse events was abstracted from the original reports on to specially designed, pretested paper forms by at least two independent

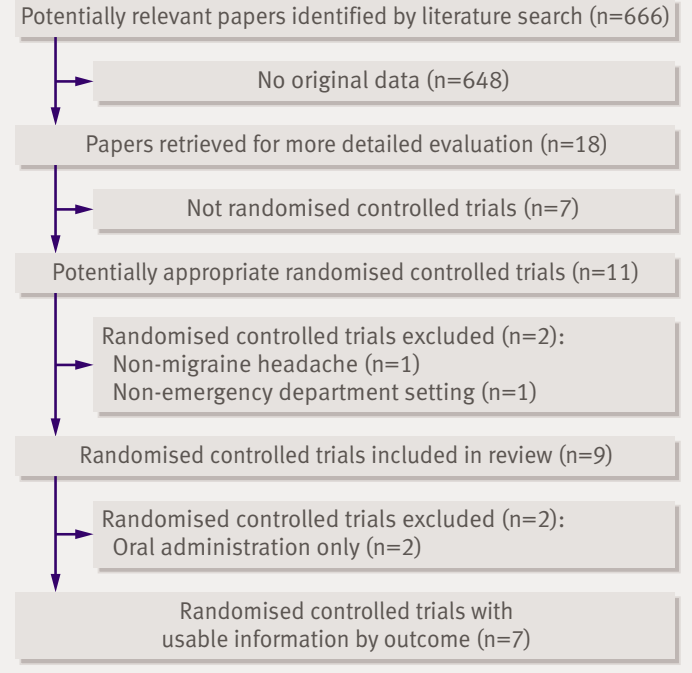

Fig 1| Flow of papers through review

reviewers (IC, MDB, and BHR). Disagreements were resolved through discussion.

We assessed the internal validity of individual trials using Jadad's scale, ${ }^{15}$ which evaluates the reported randomisation, blinding, and withdrawals in a clinical trial and assigns a score from 0 to 5 , with higher scores indicating higher quality in the conduct or reporting of the trial.

All data were entered into RevMan software (version 4.2 for Windows; IMS, Copenhagen, Denmark). We pooled the results of studies using fixed effects models, if appropriate, after consideration of heterogeneity among trials. For dichotomous variables we calculated individual and pooled statistics as relative risks with $95 \%$ confidence intervals. For continuous outcomes we calculated the mean differences reported from individual trials, and we pooled statistics as weighted mean differences with associated 95\% confidence intervals. We used $\chi^{2}$ and $\mathrm{I}^{2}$ statistics to test for heterogeneity (25\%, $50 \%$, and $75 \%$ representing low, moderate, and high heterogeneity). ${ }^{16}$

Owing to insufficient data it was not always possible to carry out anticipated subgroup comparisons and sensitivity analyses. We did not do a planned subgroup comparison of different corticosteroid agents because the included studies all used dexamethasone. We did, however, complete a priori subgroup analyses comparing intramuscular administration with intravenous administration, and random and fixed effects sensitivity analyses. In addition, we carried out retrospective subgroup analyses of dosage and duration of follow-up.

\section{RESULTS}

Overall, 666 potentially relevant abstracts were identified (fig 1). Two reviewers determined independently that 18 of these required review of the full manuscript and seven of the 18 fulfilled study eligibility criteria; thus seven trials were included in this 
review. ${ }^{\text {w1-w7 }}$ No disagreements on inclusion of trials occurred between reviewers. Five of the seven primary papers were published and two were in abstract form; however, study data were acquired through correspondence with the main author.

The included trials all used dexamethasone as the corticosteroid agent and had similar study methods. The table summarises the key features of the included trials. Overall, the quality of the studies was high as most concealed allocation from the patients and treating clinicians, were double blinded, and reported losses to follow-up.

\section{Primary outcome}

All seven studies $(\mathrm{n}=738)$ reported a lower recurrence of migraine within 24-72 hours of treatment for the original migraine headache among the dexamethasone groups than the placebo groups (fig 2). Effect sizes varied slightly among trials; however, heterogeneity was non-significant $\left(\mathrm{I}^{2}=3.4 \%\right)$. The pooled results indicated a significant reduction in recurrence rates among those treated with dexamethasone ( $v$ placebo) in addition to standard abortive care (relative risk 0.74 , $95 \%$ confidence interval 0.60 to 0.90 ). The estimated number needed to treat to prevent one recurrent headache was 9 (95\% confidence interval 6 to 25$)$.

\section{Headache scores}

Four studies $(n=455)$ reported mean differences in headache pain on a visual analogue scale from baseline to discharge from the emergency department. ${ }^{\text {w2 w3 w5 w7 }}$ Standard deviations were not available for all studies and were imputed using the largest standard deviation from the included trials. The results suggest no benefit in initial pain reduction for those treatments that included dexamethasone compared with placebo (weighted mean difference 0.37, 95\% confidence interval -0.20 to 0.94 ); however, this study pooling demonstrated moderate heterogeneity $\left(\mathrm{I}^{2}=46.2 \%\right)$. As the treating doctor had the choice of abortive therapy, numerous cointerventions were used and these were not randomised, whereas allocation to corticosteroid or placebo was randomised and concealed.

\section{Adverse events}

Six of the studies $(n=626)$ reported on specific adverse events. ${ }^{\text {w1-w3 } 35-\mathrm{w} 7}$ No significant differences were found between dexamethasone and placebo groups for restlessness, drowsiness, tingling, numbness, or swelling (fig 3). Patients treated with dexamethasone were more likely to have dizziness (relative risk $2.15,0.98$ to 4.74 ) but less likely to have nausea $(0.70,0.48$ to 1.02$)$ or “other" adverse events $(0.50,0.30$ to 0.82$)$.

\begin{tabular}{|c|c|c|c|c|c|c|c|c|c|}
\hline \multirow[b]{2}{*}{ Reference } & \multirow[b]{2}{*}{$\begin{array}{c}\text { No of } \\
\text { participants }\end{array}$} & \multirow[b]{2}{*}{ Setting } & \multirow[b]{2}{*}{ Treatment } & \multirow[b]{2}{*}{ Comparison } & \multirow[b]{2}{*}{$\begin{array}{l}\text { Follow-up } \\
\text { period } \\
\text { (hours) }\end{array}$} & \multicolumn{4}{|c|}{ Trial quality } \\
\hline & & & & & & $\begin{array}{l}\text { Blinding } \\
\text { described, } \\
\text { appropriate }\end{array}$ & $\begin{array}{l}\text { Randomisation } \\
\text { described, } \\
\text { appropriate }\end{array}$ & $\begin{array}{l}\text { Losses to } \\
\text { follow-up } \\
\text { described }\end{array}$ & $\begin{array}{l}\text { Jadad } \\
\text { score }\end{array}$ \\
\hline $\begin{array}{l}\text { Baden } \\
2006^{\mathrm{w} 2}\end{array}$ & 55 & $\begin{array}{l}\text { Emergency } \\
\text { departments of two } \\
\text { military hospitals }\end{array}$ & $\begin{array}{l}\text { Standard abortive } \\
\text { therapy and } \\
\text { dexamethasone } 10 \mathrm{mg} \\
\text { intravenously }\end{array}$ & $\begin{array}{l}\text { Standard abortive } \\
\text { therapy and placebo } \\
\text { intravenously }\end{array}$ & $48-72$ & Yes, yes & Yes, no & Yes & 4 \\
\hline Innes $1999^{w 1}$ & 98 & $\begin{array}{l}\text { Emergency } \\
\text { departments of two } \\
\text { hospitals }\end{array}$ & $\begin{array}{l}\text { Standard abortive } \\
\text { therapy and } \\
\text { dexamethasone } 24 \mathrm{mg} \\
\text { intravenously }\end{array}$ & $\begin{array}{l}\text { Standard abortive } \\
\text { therapy and placebo } \\
\text { intravenously }\end{array}$ & $48-72$ & Yes, yes & Yes, yes & Yes & 5 \\
\hline Jones $2003^{\text {w5 }}$ & 70 & Emergency department & $\begin{array}{l}\text { Standard abortive } \\
\text { therapy and } \\
\text { dexamethasone } 20 \mathrm{mg} \\
\text { intravenously or } \\
\text { intramuscularly }\end{array}$ & $\begin{array}{l}\text { Standard abortive } \\
\text { therapy and placebo } \\
\text { intravenously or } \\
\text { intramuscularly }\end{array}$ & 48 & Yes, yes & Yes, yes & Yes & 5 \\
\hline Rowe $2008^{\text {w7 }}$ & 126 & $\begin{array}{l}\text { Emergency } \\
\text { departments of four } \\
\text { hospitals }\end{array}$ & $\begin{array}{l}\text { Standard abortive } \\
\text { therapy and } \\
\text { dexamethasone } 15 \mathrm{mg} \\
\text { intravenously }\end{array}$ & $\begin{array}{l}\text { Standard abortive } \\
\text { therapy and placebo } \\
\text { intravenously }\end{array}$ & $48-72$ & Yes, yes & Yes, yes & Yes & 5 \\
\hline $\begin{array}{l}\text { Friedman } \\
2007^{\text {w3 }}\end{array}$ & 205 & $\begin{array}{l}\text { Emergency } \\
\text { departments of four } \\
\text { hospitals }\end{array}$ & $\begin{array}{l}\text { Metoclopramide } 20 \mathrm{mg} \\
\text { intravenously and } \\
\text { diphenhydramine } \\
25 \mathrm{mg} \text { intravenously } \\
\text { and dexamethasone } \\
10 \mathrm{mg} \text { intravenously }\end{array}$ & $\begin{array}{l}\text { Metoclopramide } 20 \mathrm{mg} \\
\text { intravenously and } \\
\text { diphenhydramine } \\
25 \mathrm{mg} \text { intravenously } \\
\text { and placebo } \\
\text { intravenously }\end{array}$ & 24 & Yes, yes & Yes, yes & Yes & 5 \\
\hline $\begin{array}{l}\text { Fiessler } \\
2006^{\text {w4 }}\end{array}$ & 91 & Emergency department & $\begin{array}{l}\text { Standard abortive } \\
\text { therapy and } \\
\text { dexamethasone } 10 \mathrm{mg} \\
\text { intravenously }\end{array}$ & $\begin{array}{l}\text { Standard abortive } \\
\text { therapy and placebo } \\
\text { intravenously }\end{array}$ & $24-48$ & Yes, yes & Yes, no & Yes & 4 \\
\hline $\begin{array}{l}\text { Donaldson } \\
2006^{w 6}\end{array}$ & 99 & $\begin{array}{l}\text { Multiple emergency } \\
\text { departments }\end{array}$ & $\begin{array}{l}\text { Standard abortive } \\
\text { therapy and } \\
\text { dexamethasone } 24 \mathrm{mg} \\
\text { intravenously }\end{array}$ & $\begin{array}{l}\text { Standard abortive } \\
\text { therapy and placebo } \\
\text { intravenously }\end{array}$ & 72 & Yes, yes & Yes, yes & Yes & 5 \\
\hline
\end{tabular}




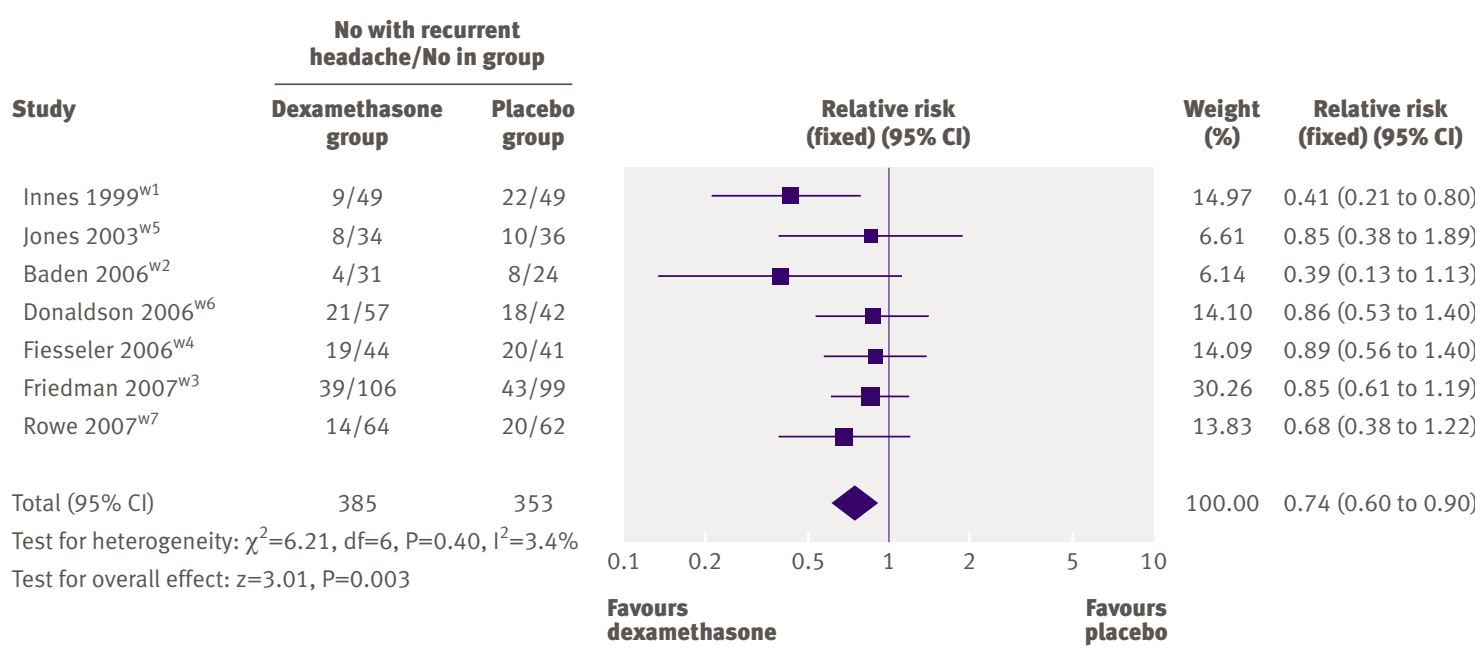

Fig 2 | Effectiveness of dexamethasone plus standard abortive therapy for recurrent migraine headache compared with placebo plus standard abortive therapy

\section{Subgroups}

Forty seven patients in one study ${ }^{\mathrm{w} 5}$ received treatment intramuscularly, allowing for an a priori subgroup comparison with those who received treatment intravenously in the same and other studies $(n=592)$. For the primary outcome of recurrent migraine, no significant differences were found between intramuscular administration (relative risk $0.59,0.19$ to 1.81 ) and intravenous administration $(0.75,0.61$ to 0.91$)$.

Retrospective subgroup analyses investigated differences in primary outcome according to dosage of dexamethasone and duration of follow-up. Studies that used less than $15 \mathrm{mg}(\mathrm{n}=3)$ of dexamethasone reported a weaker treatment effect (relative risk $0.80,0.62$ to 1.04 ) than those that used $15 \mathrm{mg}$ or more $(0.67,0.50$ to $0.91)$; the difference between these two subgroups was, however, not significant $\left(\chi^{2}=0.74 ; \mathrm{df}=1 ; \mathrm{P}=0.39\right)$. Stronger evidence suggested that studies with longer follow-up periods showed a stronger treatment effect with dexamethasone. Studies with a follow-up period of 48 hours or less showed a weaker treatment effect (relative risk $0.86,0.66$ to 1.11 ) than those with a followup period of more than 48 hours $(0.61,0.45$ to 0.84 ); moreover, the difference between these two subgroups was significant $\left(\chi^{2}=4.33 ; \mathrm{df}=1 ; \mathrm{P}=0.037\right)$.

\section{Sensitivity analyses}

The primary results were not influenced by the use of fixed effect models compared with random effect models, high quality studies compared with low quality studies, or odds ratios compared with relative risks.

\section{DISCUSSION}

This systematic review summarises the available evidence for the use of parenteral corticosteroids in treating acute severe migraine headaches. Using a comprehensive and exhaustive search strategy we identified seven high quality randomised controlled trials evaluating the use of dexamethasone in acute migraine. Our results suggest that, when added to standard abortive therapy, dexamethasone reduces the recurrence of headache within 72 hours, and that only nine patients need to be treated to prevent one recurrent headache. Dexamethasone is readily available and familiar to doctors in most parts of the world, ${ }^{17}$ and when used as a single parenteral dose - as in all of the included trials - it is well tolerated, causing only minor side effects. This review does not support the use of corticosteroids for immediate relief of acute migraine. Evidence from four included studies suggests that dexamethasone does not significantly reduce pain scores before discharge from an emergency department, but that its potential benefits are more related to a reduction in recurrence of headaches.

Previous studies have found that doctors consider the recurrence of headaches to be a less important outcome of treatment, ${ }^{18}$ but patients view it as a very important outcome - second only to complete relief from headache. ${ }^{19}$ When a willingness to pay model was used, patients considered lower relapse rates to be the outcome of greatest importance,$^{20}$ suggesting that treatments to reduce headache recurrence should be a high priority in migraine research.

The trials reviewed show apparent benefit of corticosteroid treatment for acute severe migraine headache, but several problems remain unresolved. For example, because of the relatively small number of patients available for subgroup analysis we could not identify the characteristics of patients most likely to benefit from this treatment, although two studies suggested that treatment effect is related to duration of headache and that dexamethasone may be more useful in patients with prolonged severe headaches. ${ }^{\text {w3 }}$ ${ }^{\text {w7 }}$ In addition, we could not clarify the relation between different abortive agents provided in the emergency department and headache recurrence rates, nor the potential interaction between these different abortive agents and dexamethasone. For example, previous evidence shows that opioids may be less effective than other agents as abortive agents and associated with 


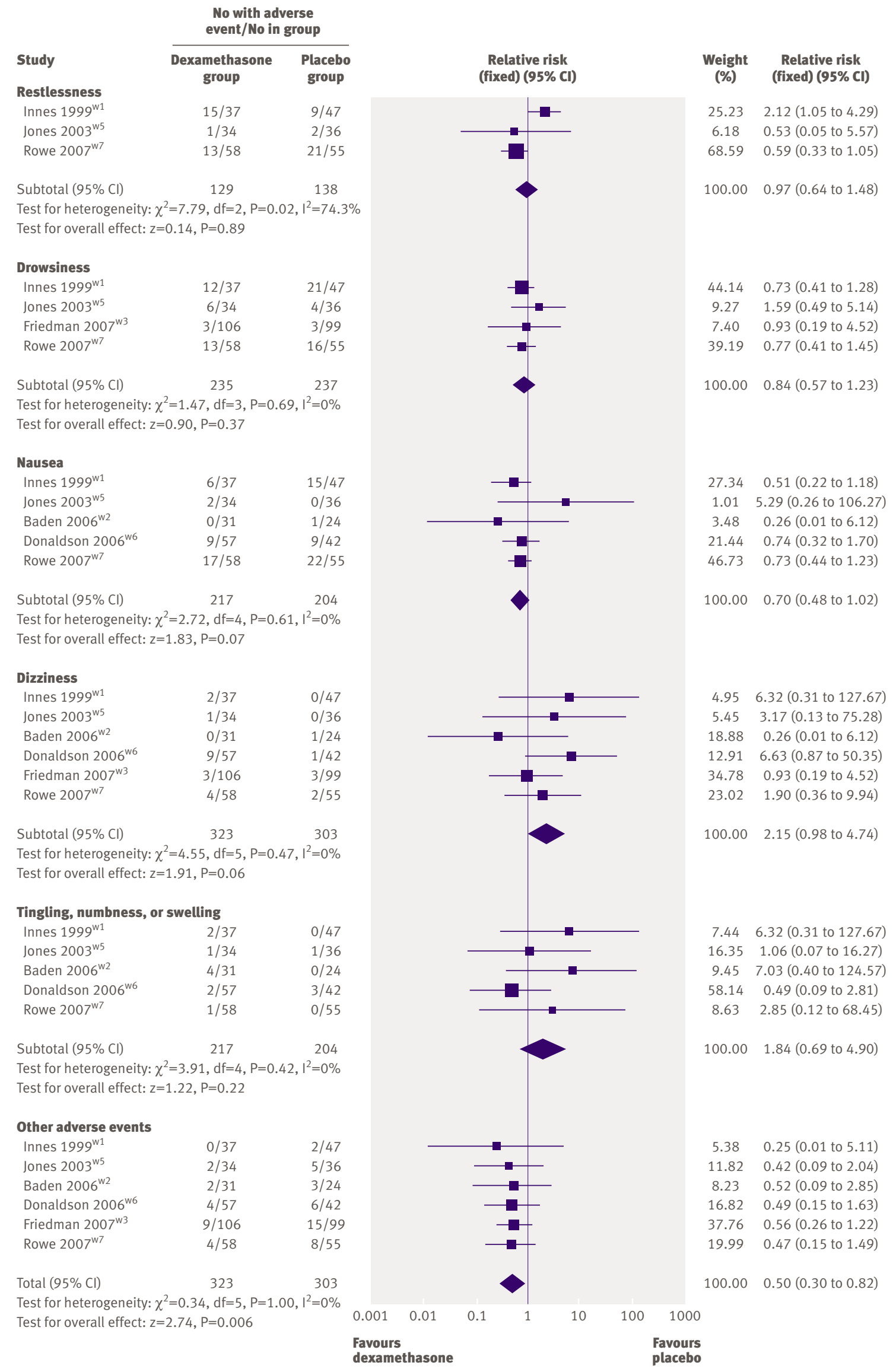

Fig 3 | Adverse events after treatment of migraine headaches using dexamethasone plus standard abortive therapy compared with placebo plus standard abortive therapy 


\section{WHAT IS ALREADY KNOWN ON THIS TOPIC}

Recurrent headache is common within 24-72 hours after

treatment for acute severe migraine headache

Inflammation may play a part in migraine, and

corticosteroids might reduce the rate of recurrent headache

\section{WHAT THIS STUDY ADDS}

Dexamethasone reduces rates of recurrent headache after treatment for acute severe migraine headache

Evidence is lacking that dexamethasone provides

immediate pain relief from migraine

higher relapse rates. ${ }^{21}$ Consequently, the differing use of primary abortive agents across studies may be an important confounding factor. One trial suggested that more severe residual pain in the emergency department was associated with a greater risk of headache relaps ${ }^{\mathrm{w} 7}$; therefore future study of abortive agents and relapse rates is warranted.

Finally, although we did not evaluate the effectiveness of oral corticosteroids, it is possible that they have beneficial effects similar to those delivered parenterally. For example, in other diseases where corticosteroids have been shown to be effective, such as in asthma, meta-analyses failed to show a difference between oral and intravenous routes of delivery ${ }^{22}$ Caution is advised in translating this to the population with acute severe migraine as two recent randomised placebo controlled trials using oral dexamethasone ${ }^{23}$ and prednisone $e^{24}$ failed to show a benefit of oral corticosteroids when added to abortive therapy for the prevention of recurrent migraine headache. Recent evidence, however, suggests that oral non-steroidal anti-inflammatory drugs (naproxen sodium $500 \mathrm{mg}$ ) may also be effective in the prevention of recurrent migraine headache in a primary care population..$^{25}$

\section{Limitations}

This review has some limitations. Firstly, despite the use of appropriate and similar criteria to assess patient eligibility across included studies, it is likely that people with non-migraine headache were enrolled. This reflects the fact that migraine is a clinical diagnosis, that International Headache Society diagnostic criteria lack perfect specificity, and that clinical overlap exists between migraine headache and severe non-migraine headaches. In addition, this reflects the diagnosis and management of migraine headaches in the real world. Importantly, the studies reviewed were carried out in emergency departments and headache clinics; the results are likely to be generalisable to similar settings but may not be generalisable to primary care or other outpatient settings.

Secondly, although it is likely that single dose dexamethasone is relatively safe, as described by the authors of the primary studies, the studies did not follow patients beyond 72 hours and may have missed uncommon or delayed adverse events. Given the frequency of migraine headaches, studies may be required to assess the likelihood of rare or long term adverse events. For example, prolonged oral corticosteroid therapy has been linked to loss of bone mineral density and increased rates of fractures. ${ }^{26}$ Additionally, patients with migraine headaches may require repeated visits to the emergency department and could be exposed to multiple doses of steroids and their side effects.

Recent evidence suggests that publication bias is less pervasive in the emergency department literature ${ }^{27}$; however, trials reporting negative findings are less likely to be published and more likely to be excluded from systematic review, potentially biasing the study conclusions. We believe that our comprehensive search strategy, which included a hand search of recent conference proceedings to identify unpublished trials, minimised any such bias. In fact we included two unpublished trials ${ }^{\mathrm{w} 4 \mathrm{w} 5}$ and all but one trial ${ }^{\mathrm{w} 1}$ with "negative" findings in our analysis. It is unlikely that publication bias influenced our conclusions.

Finally, selection bias is always possible. To minimise the likelihood of such bias, two independent reviewers screened all abstracts and primary manuscripts by using standardised eligibility criteria.

\section{Conclusion}

When added to standard abortive migraine therapy, single dose parenteral dexamethasone is associated with a $26 \%$ relative reduction in recurrent headache (NNT=9) occurring within 72 hours. Further research is required to determine whether specific patient subgroups are more likely to benefit from this treatment.

We thank the department of emergency medicine at the University of Alberta; ICN Pharmaceuticals and Faulding Pharmaceutical for responding to our requests for unpublished data; and $\mathrm{A}$ Bastani, a corresponding study author. Data from this study were reported at the Canadian Association of Emergency Physicians annual scientific meeting, Winnipeg, MB, Canada, 14-17 June, 2003

Contributors: IC conceived the project, carried out the searches,

coordinated the reviewers, collected and extracted the data, and prepared the manuscript. BWF interpreted and provided data. MDB contributed to the protocol and collected and extracted data. GDI contributed to the protocol, selected articles, and reviewed the manuscript. EG and TER contributed to the protocol and selected articles. BHR conceived the project, contributed to the protocol, coordinated the review, selected articles, collected and extracted data, prepared the manuscript, and secured project funding. All authors helped revise the manuscript. IC and BHR are guarantors for the paper.

Funding: BHR is supported by the 21st century Canada research chairs programme through the government of Canada. The Canadian Association of Emergency Physicians Research Consortium provided partial support for this study.

Competing interests: GDI, BWF, and BHR are primary authors of included trials. MDB and IC are coauthors of included trials.

Ethical approval: Not required.

Provenance and peer review: Not commissioned; externally peer reviewed.

1 Stewart WF, Shechter A, Rasmussen BK. Migraine prevalence. A review of population-based studies. Neurology 1994:44:S17-23.

2 Osterhaus JT, Gutterman DL, Plachetka JR. Healthcare resource and lost labour costs of migraine headache in the US.

Pharmacoeconomics 1992;2:67-76.

3 Vinson DR. Treatment patterns of isolated benign headache in US emergency departments. Ann Emerg Med 2002;39:215-22. 
4 Snow V, Weiss K, Wall EM, Mottur-Pilson C. Pharmacologic management of acute attacks of migraine and prevention of migraine headache. Ann Intern Med 2002;137:840-9.

5 Silberstein SD. Practice parameter: evidence-based guidelines for migraine headache (an evidence-based review): report of the Quality Standards Subcommittee of the American Academy of Neurology. Neurology 2000;55:754-62.

6 Pryse-Phillips WE, Dodick DW, Edmeads JG, Gawel MJ, Nelson RF, Purdy RA, et al. Guidelines for the diagnosis and management of migraine in clinical practice. Canadian Headache Society. CMA 1997;156:1273-87.

7 Moore KL, Noble SL. Drug treatment of migraine: part I. Acute therapy and drug-rebound headache. Am Fam Physician 1997;56:2039-48, 2051-4.

8 Raskin NH. Acute and prophylactic treatment of migraine: practical approaches and pharmacologic rationale. Neurology 1993;43:S39-42.

9 Ducharme J, Beveridge RC, Lee JS, Beaulieu S. Emergency management of migraine: is the headache really over? Acad Emerg Med 1998;5:899-905.

10 Bond K, Ospina MB, Blitz S, Afilalo M, Campbell SG, Bullard M, et al. Frequency, determinants and impact of overcrowding in emergency departments in Canada: a national survey. Healthc Q 2007;10:32-40.

11 Welch KM. Contemporary concepts of migraine pathogenesis. Neurology 2003;61:S2-8.

12 Hamel E. Current concepts of migraine pathophysiology. Can J Clin Pharmacol 1999;6(suppl A):9A-14A.

13 Goadsby PJ. Current concepts of the pathophysiology of migraine. Neurol Clin 1997;15:27-42

14 Colman I, Innes GD, Brown MD, Grafstein E, Roberts TE, Rowe BH Parenteral corticosteroids for acute migraine (protocol for a Cochrane Review). Cochrane Library. Oxford: Update Software, 2003.

15 Jadad AR, Moore RA, Carroll D, Jenkinson C, Reynolds DJ, Gavaghan DJ, et al. Assessing the quality of reports of randomized clinical trials: is blinding necessary? Control Clin Trials 1996;17:1-12.

16 Higgins JP, Thompson SG, Deeks JJ, Altman DG. Measuring inconsistency in meta-analyses. BMJ 2003;327:557-60.
17 Ausejo M, Saenz A, Pham B, Kellner JD, Johnson DW, Moher D, et al. The effectiveness of glucocorticoids in treating croup: meta-analysis. BMJ 1999;319:595-600

18 Lipton RB, Hamelsky SW, Dayno JM. What do patients with migraine want from acute migraine treatment? Headache 2002;42(suppl 1):3-9.

19 Lipton RB, Stewart WF. Acute migraine therapy: do doctors understand what patients with migraine want from therapy? Headache 1999;39(suppl 2):S20-6.

20 Lenert LA. Use of willingness to pay to study values for pharmacotherapies for migraine headache. Med Care 2003;41:299-308

21 Colman I, Rothney A, Wright SC, Zilkalns B, Rowe BH. Use of narcotic analgesics in the emergency department treatment of migraine headache. Neurology 2004;62:1695-700.

22 Rowe BH, Spooner C, Ducharme FM, Bretzlaff JA, Bota GW. Early emergency department treatment of acute asthma with systemic corticosteroids. Cochrane Database Syst Rev 2001:CD002178.

23 Kelly AM, Kerr D, Clooney M. Impact of oral dexamethasone versus placebo after ED treatment of migraine with phenothiazines on the rate of recurrent headache: a randomised controlled trial. Emerg Med / 2008;25:26-9.

24 Fiessler F, Shih R, Silverman M, Eskin B, Clement M, Szucs $P$, et al. Prednisone for migraine headaches: an emergency department randomized double-blind placebo-controlled trial. Acad Emerg Med 2007;14(suppl 1):S71.

25 Brandes JL, Kudrow D, Stark SR, O'Carroll CP, Adelman JU, O’Donnell FJ, et al. Sumatriptan-naproxen for acute treatment of migraine: randomized trial. JAMA 2007;297:1443-54.

26 Van Staa TP, Leufkens HG, Cooper C. The epidemiology of corticosteroid-induced osteoporosis: a meta-analysis. Osteoporos Int 2002;13:777-87.

27 Ospina MB, Kelly K, Klassen TP, Rowe BH. Publication bias of randomized controlled trials in emergency medicine. Acad Emerg Med 2006;13:102-8.

Accepted: 11 April 2008 